\title{
Perfil das parturientes e seus recém-nascidos atendidos em um hospital-escola do Noroeste do Estado do Paraná
}

\author{
PROFILE OF PARTURIENTS AND THEIR NEWBORN BABIES RECEIVING CARE \\ IN A UNIVERSITY HOSPITAL IN NORTHWESTERN PARANÁ \\ PERFIL DE LAS PARTURIENTAS Y SUS RECIÉN NACIDOS ATENDIDOS EN UN \\ HOSPITAL ESCUELA DEL NOROESTE DEL ESTADO DE PARANÁ
}

Gisele Ferreira da Silva1, Sandra Marisa Pelloso²

\begin{abstract}
RESUMO
O estudo teve como objetivo traçar o perfil de parturientes e seus recém-nascidos atendidos em um hospital-escola, a partir do Sistema de Informações sobre Nascidos Vivos - SINASC. Para isso, foi realizado um estudo descritivo exploratório que identificou as características de todas parturientes e seus recém-nascidos atendidos no Hospital Universitário de Maringá - HUM no ano de 2006. Observou-se que $27,1 \%$ das parturientes eram adolescentes, 68,4\% não tinham companheiro, $59,3 \%$ residiam fora de Maringá, 63,6\% realizaram sete ou mais consultas de pré-natal. A proporção de parto cesárea foi de $50,4 \%$. Os recém-nascidos, em sua maioria, apresentaram boa vitalidade ao nascer, embora $25,5 \%$ eram prétermos e $23,6 \%$ baixo peso ao nascer. Com os resultados, foi possível traçar o perfil das parturientes e seus recém-nascidos atendidos no HUM, demonstrar a possibilidade de utilização do SINASC como um dos instrumentos de avaliação em saúde em unidade terciária e propor intervenções capazes de prevenir a morbimortalidade materno-infantil.
\end{abstract}

\section{DESCRITORES}

Sistemas de informação.

Saúde materno-infantil.

Avaliação em saúde.

\begin{abstract}
The study had the purpose to outline the profile of parturients and their newborn babies receiving care in a school hospital, based on the Live Birth Information System - Sistema de Informações sobre Nascidos Vivos - SINASC. For so, a descriptive, exploratory study was elaborated, identifying the characteristics of all the parturients and their newborns receiving care at Hospital Universitário de Maringá - HUM in 2006. It was observed that $27.1 \%$ of the parturients were adolescents, $68.4 \%$ had no partner, 59.3\% lived outside Maringá, and 63.6\% had seven or more pre-natal appointments. The ratio of cesarian sections was $50.4 \%$. The newborns, mostly, had good vitality at birth, although $25.5 \%$ were preterms and $23.6 \%$ had low weight at birth. With the results, it was possible to outline the profile of the parturients and their newborns receiving care at the HUM, show the possibility of using the SINASC as an instrument of health evaluation in tertiary units and propose interventions capable of preventing maternal-child morbimortality.
\end{abstract}

\section{KEY WORDS}

Information systems.

Maternal and child health.

Health evaluation.

\section{RESUMEN}

El estudio tuvo como objetivo trazar el perfil de parturientes y sus recién nacidos atendidos en un hospital escuela, a partir del Sistema de Informaciones sobre Nacidos Vivos - SINASC. Para lo cual, fue realizado un estudio descriptivo y exploratorio que identificó las características de todas las parturientes y sus recién nacidos atendidos en el Hospital Universitario de Maringá - HUM en el año de 2006. Se observó que 27,1\% de las parturientes eran adolescentes, $68,4 \%$ no tenían compañero, 59,3\% residían fuera de Maringá, 63,6\% realizaron siete o más consultas de prenatal. La pro-porción de parto cesárea fue de 50,4\%. Los recién nacidos, en su mayoría, presentaron una buena vitalidad al nacer, a pesar de que $25,5 \%$ eran prematuros y $23,6 \%$ de bajo peso al nacer. Con los resultados, fue posible trazar el perfil de las parturientas y de sus recién nacidos, atendidos en el HUM, y demostrar la posibilidad de utilizar el SINASC como uno de los instrumentos de evaluación de la salud en una unidad terciaria y proponer intervenciones capaces de prevenir la morbimortalidad maternoinfantil.

\section{DESCRIPTORES}

Sistemas de información. Salud materno-infantil. Evaluación en salud.

${ }^{1}$ Mestre em Enfermagem. Enfermeira do Hospital Universitário de Maringá. Maringá, PR, Brasil. gifsnurse@yahoo.com.br. ${ }^{2}$ Enfermeira. Doutora em Enfermagem. Professora da Universidade Estadual de Maringá. Maringá, PR, Brasil. smpelloso@uem.br.

$\begin{array}{rlr}\text { Português / Inglês } & \text { Recebido: 14/01/2008 } & \text { Rev Esc Enferm USP } \\ \text { www.scielo.br/reeusp } & \text { Aprovado: 31/07/2008 } & \text { w3(1):95-102 } \\ \text { www.ee.usp.br/reeusp/ }\end{array}$




\section{INTRODUÇÃO}

O conhecimento sobre as características dos nascimentos em um determinado local e período é de fundamental importância, para possibilitar a elaboração de indicadores demográficos e epidemiológicos que podem representar a realidade da comunidade para o planejamento e implementação de estratégias efetivas de melhorias das condições de saúde. A cada ano, nascem, no Brasil, aproximadamente, 3.100.000 crianças. A região sul, em 2005, apresentou $12 \%$ dos nascimentos do Brasil; dos Estados da região sul, o Paraná com 40,8\% apresentou o maior número de nascimentos da região sul e o município de Maringá representou $3,5 \%$ dos nascimentos do Paraná(1).

No Brasil, há várias fontes de informação sobre o número de nascimentos, e entre as principais fontes de informação, é citado o Instituto Brasileiro de Estatística e Geografia - IBGE com os censos demográficos, as Pesquisas Nacionais por Amostra de Domicílios - PNAD e o Sistema de Informação sobre Nascidos Vivos - SINASC. A existência de sistemas de informações é fundamental para viabilizar orientações e ações que estão sendo e/ou deverão ser desenvolvidas nos serviços de saúde(2).

Os Sistemas de Informação em Saúde - SIS, no Brasil, e a descentralização dos serviços de saúde foram criados como uma das estratégias do Sistema Único de Saúde - SUS para melhorias da situação de saúde da população. Dentre os SIS, o SINASC é um dos mais utilizados pelos municípios e Estados por servir de fonte para cálculo das proporções de nascidos vivos com baixo peso ao nascer, nascidos vivos de mães com quatro ou mais e sete ou mais consultas de pré-natal, e de partos cesáreos, coeficiente de mortalidade infantil, neonatal e neonatal tardia, razão de mortalidade materna, entre outros que compõem os indicadores de monitoramento e avaliação do Pacto pela Saúde, criado para redefinir as responsabilidades de cada gestor em função das necessidades de saúde de cada população na busca da equidade social.

O SINASC é uma fonte de informação sobre nascimentos, implantado em escala nacional pelo Ministério da Saúde desde 1990, em resposta à necessidade de um banco de dados que disponibilizasse, além da contagem do número de nascimentos ocorrido no ano, informações sobre características das mães, da gestação, do parto e do recém-nascido(3). A implantação do SINASC ocorreu de forma gradativa no Brasil; no Paraná, ocorreu a partir de novembro e dezembro de $1993^{(2)}$, e sua utilização, no Paraná, foi ampliada com a sua disponibilidade em nível municipal com supervisão do Estado, tornando possível sua utilização em nível local. Atualmente, o SINASC está implantado em todos os Estados e disponível na página eletrônica do Departamento de Informação e Informática do SUS/ Ministério da Saúde.
O SINASC utiliza como fonte de dados a Declaração de Nascidos Vivos - DNV, documento de emissão obrigatória pelos Cartórios de Registro Civil nos serviços onde ocorreu o parto, e nos casos de partos domiciliares ou em outros locais onde não houve assistência por profissionais da saú$\mathrm{de}^{(3)}$. A partir do preenchimento da DNV, é possível obter informações fundamentais para o planejamento das ações do cuidado obstétrico, para avaliação de ações em saúde, e para construção de indicadores demográficos e de saúde na área materno-infantil.

A população brasileira não se enquadra em um único padrão social e educacional, e com a grande diferença em cada região em relação ao atendimento de saúde, não é possível generalizar o tipo de atendimento obstétrico prestado pelas várias instituições. Dependendo da região em que se encontram as atividades de atendimento à mulher, existem características peculiares ligadas aos aspectos demográficos, culturais, sociais e econômicos que determinam padrões diferentes de comportamento da população feminina ${ }^{(4)}$.

Ao considerar instituição de referência para acompanhamento de gestações de risco, há o interesse em traçar o perfil da clientela assistida, para reconhecer suas características e identificar fatores de risco e intercorrências que poderiam ser evitadas ou tratadas ${ }^{(5)}$. Mediante as considerações expostas, acredita-se serem oportunos os estudos que caracterizam a população atendida em cada instituição. E que é função das instituições universitárias, de ensino e pesquisa, elaborarem modelos de assistência que possam ser reproduzidos em maior escala, em termos populacionais, segundo a especificidade de cada grupo.

Diante destes dados, é fundamental conhecer quem são as parturientes e seus recém-nascidos atendidos no contexto de um hospital-escola. A princípio, espera-se que com o perfil das parturientes e seus recémnascidos, o SINASC possa ser utilizado como um dos instrumentos de avaliação em unidade terciária e intervenha em ações capazes de prevenir a morbimortalidade.

No contexto dos desafios e perspectivas vivenciadas pelos hospitais universitários do Brasil, talvez o passo mais importante a ser dado seja o fortalecimento da sua sustentabilidade organizacional, que incluem entre outros o desenvolvimento de sistemas de indicadores de avaliação e gestão(6).

\section{OBJETIVO}

O presente estudo teve como objetivo traçar o perfil de parturientes e seus recém-nascidos ( $R N$ ) atendidos em um hospital-escola, demonstrando a potencialidade de utilização do SINASC como um dos instrumentos de avaliação em saúde materno-infantil em unidade terciária. 


\section{MÉTODO}

Foi realizado um estudo descritivo exploratório que, a partir do SINASC, identificou as características de todas parturientes e seus RN atendidos em um hospital-escola no ano de 2006.

O hospital-escola estudado foi o Hospital Universitário de Maringá - HUM, localizado na região noroeste do Estado do Paraná, referenciado no atendimento ao parto às gestantes de alto risco procedentes dos 29 municípios da 15a Regional de Saúde - RS, e de gestantes de baixo e alto risco da área de abrangência de quatro Unidades Básicas de Saúde do município de Maringá. É um hospitalescola com rotinas implantadas e apoiadas pelo Ministério da Saúde no que se refere ao atendimento à gestação e parto. O HUM foi inaugurado em janeiro de 1989, e, em 1993, a Clínica de Ginecologia e Obstetrícia iniciou suas atividades. Encontra-se credenciado junto ao SUS, caracterizando-se como instituição de caráter público, está designado como hospital-escola e em razão de sua capacidade operacional ativa está classificado como hospital de porte tipo II.

O perfil das parturientes e dos seus RN foi identificado por meio de variáveis sociodemográficas (idade materna, estado civil, escolaridade, ocupação e município de residência), variáveis obstétricas (filhos nascidos vivos, filhos nascidos mortos, idade gestacional, tipo de gravidez, número de consultas de pré-natal, tipo de parto) e variáveis do $\mathrm{RN}$ (sexo, Apgar 10 minuto, Apgar 5o minuto, raça/cor, peso, malformação congênita/ anomalia cromossômica).

Para a variável malformação congênita/anomalia cromossômica, foi utilizado o termo malformação congênita, a variável estado civil foi denominada por situação conjugal, por indicar um termo mais adequado para a presença de apoio econômico de uma situação estável e pelo apoio psicossocial de ter um companheiro. E para a variável número de consultas de pré-natal foi considerado $\geq 7$ número adequado de consulta, que de acordo com as instruções dos indicadores para a pactuação é específica para os municípios em que a proporção de nascidos vivos de mães de quatro ou mais consultas de pré-natal superou $90 \%$ do ano anterior, situação indicada neste estudo uma vez que apresentou $91,1 \%$ de nascidos vivos de mães de quatro ou mais consultas de pré-natal.

Os dados foram disponibilizados pela 15a RS pelo Programa Tabwin, após foram tabulados e processados eletronicamente pelo programa Statistica 6.0 (STATSOFT). Os dados obtidos são considerados preliminares, uma vez que na sua coleta não estavam disponíveis on-line, porém acredita-se na sua fidedignidade por já terem sido avaliados pela digitação municipal e o total de nascimentos serem compatíveis com o livro de registro do HUM.

Foram analisadas todas DNV presentes no SINASC-2006 referentes aos nascidos no HUM, e utilizadas frequências absolutas e relativas para todas variáveis estudadas, evidenciando os resultados em tabelas e discussão dos resultados, a partir de dados do Ministério da Saúde e demais referências para análise da temática. Utilizou-se a análise multivariada de correspondência múltipla entre as variáveis município de residência da mãe, tipo de parto, número de consultas de pré-natal e peso ao nascer que são indicadores no Pacto pela Saúde, para visualizar a associação entre as variáveis utilizadas como indicadores.

O projeto de pesquisa foi aprovado pelo Comitê Permanente de Ética em Pesquisa envolvendo Seres Humanos da Universidade Estadual de Maringá - UEM, conforme as determinações da Resolução n. 196/96 do ConseIho Nacional de Pesquisa, sob o Parecer $n^{\circ}$. 008/2007. Como o estudo tem base em dados secundários, foi solicitado dispensa do Termo de Consentimento Livre e Esclarecido - TCLE.

\section{RESULTADOS}

O preenchimento da DNV, normalmente, é realizado pelas técnicas de enfermagem do Setor Obstétrico do HUM. Após seu preenchimento, é recolhida pela funcionária da Secretaria Municipal de Saúde para digitação no SINASC e encaminhada para a Secretaria de Estado da Saúde - SESA por meio da 15a Regional de Saúde.

O número de nascidos vivos no HUM, no ano de 2006, foi de 760 , representando aproximadamente $13 \%$ dos nascimentos ocorridos no município de Maringá.

Foram encontradas poucas variáveis sem informação, ou seja, as variáveis em branco ou com informação ignorada, que variaram de $0,1 \%$ para sexo do $\mathrm{RN}$ a $0,7 \%$ para ocupação da mãe.

A Tabela 1 apresenta as variáveis sociodemográficas referentes às parturientes. Em relação à idade, foi observado predomínio na faixa etária de 20-34 anos com $63,5 \%$, na sequência a faixa etária $<20$ anos com $27,1 \%$. Quanto à situação conjugal, 68,4\% não tinham companheiro, e a maioria apresentou pouca escolaridade, sendo $63,6 \%$ com quatro a 11 anos de estudo, $27,0 \%$ com até três anos de estudo e apenas 9,4\% com igual ou maior a 12 anos de estudo. Acrescentando à condição social das parturientes, foi verificado que somente $33,8 \%$ tinham ocupações remuneradas, e as demais $62,7 \%$ eram do lar e 3,5\% eram estudantes. A análise das parturientes, segundo o município de residência, apresenta maior porcentagem para domicílios fora de Maringá com 59,3\% e 40,7\% para moradoras do município de Maringá. Do total de parturientes não-residentes em Maringá, a maioria é moradora de Sarandi (11\%) e de Paiçandu (6\%). As demais parturientes são moradoras de outros municípios, que representaram valores menores que $1 \%$ até $5 \%$. 
Tabela 1- Perfil sociodemográfico das parturientes atendidas no Hospital Universitário de Maringá - Maringá, PR - 2006

\begin{tabular}{|c|c|c|}
\hline Variável & $\mathbf{N}$ & $\%$ \\
\hline \multicolumn{3}{|l|}{ Idade (anos) } \\
\hline$<20$ & 206 & 27,1 \\
\hline $20-34$ & 483 & 63,5 \\
\hline$\geq 35$ & 71 & 9,4 \\
\hline \multicolumn{3}{|l|}{ Situação conjugal* } \\
\hline Com companheiro & 239 & 31,6 \\
\hline Sem companheiro & 517 & 68,4 \\
\hline \multicolumn{3}{|l|}{ Escolaridade (anos)* } \\
\hline$\leq 3$ & 204 & 27,0 \\
\hline $4-11$ & 481 & 63,6 \\
\hline$\geq 12$ & 71 & 9,4 \\
\hline \multicolumn{3}{|l|}{ Ocupação** } \\
\hline Sem remuneração & 499 & 66,2 \\
\hline Com remuneração & 255 & 33,8 \\
\hline \multicolumn{3}{|l|}{ Residência } \\
\hline Maringá & 309 & 40,7 \\
\hline Outros municípios & 451 & 59,3 \\
\hline
\end{tabular}

Na Tabela 2, estão descritas as variáveis obstétricas referentes às parturientes. Em relação ao número de filhos nascidos vivos, em gestações anteriores, não houve diferença significativa entre as categorias de primíparas $(50,4 \%)$ ou multíparas (49,6\%). Já para o número de filhos nascidos mortos em gestações anteriores foi expressiva a categoria com nenhum filho nascido morto (91,0\%). A duração da gestação demonstrou que a maioria $(73,8 \%)$ ocorreu entre 37 a 41 semanas, considerada mais adequada para favorecer as condições de saúde do recém-nascido(7). Observouse que $25,5 \%$ das gestações não chegaram a termo. 0 tipo de gravidez, em sua maioria, foi única com 93,2\%. A maioria das parturientes realizou sete ou mais consultas de prénatal (63,6\%). A proporção de parto cesárea foi de 50,4\%.

Tabela 2 - Perfil obstétrico das parturientes atendidas no Hospital Universitário de Maringá - Maringá, PR - 2006

\begin{tabular}{lrr}
\hline Variável & N & $\mathbf{\%}$ \\
\hline Filhos nascidos vivos & 383 & 50,4 \\
$\quad$ Primípara & 377 & 49,6 \\
$\quad$ Multípara & & \\
Filhos nascidos mortos & 692 & 91,0 \\
$\quad$ Nenhum & 68 & 9,0 \\
$\quad \geq 1$ & & \\
$\quad$ Duração da gestação(semanas) & 194 & 25,5 \\
$\quad<37$ & 561 & 73,8 \\
37-41 & 5 & 0,7 \\
$\quad \geq 42$ & & \\
Tipo gravidez & 708 & 93,2 \\
$\quad$ Unica & 52 & 6,8 \\
$\quad$ Múltipla & & \\
Número consultas de pré-natal* & 275 & 36,4 \\
$\quad<7$ & 480 & 63,6 \\
$\quad \geq 7$ & & \\
Tipo de parto & 377 & 49,6 \\
$\quad$ Normal & 383 & 50,4 \\
$\quad$ Cesárea
\end{tabular}

* excluídos cinco casos com informação ignorada
A Tabela 3 apresenta dados referentes aos nascidos vivos. Os nascidos vivos, em sua maioria (51,5\%), eram do sexo masculino. Com relação ao índice de Apgar, que contém indicadores para avaliar as condições de nascimento do RN, a grande maioria apresentou boa vitalidade (Apgar $\geq 7)^{(7)}$ no 1 을 5 o minuto com $86,0 \%$ e $96,1 \%$, respectivamente. A maioria dos nascidos (76,4\%) pesou $2.500 \mathrm{~kg}$ ou mais, embora $23,6 \%$ tenham apresentado baixo peso ao nascer. No resultado da raça/cor dos nascidos vivos, 76,7\% eram brancos e $23,3 \%$ eram para as demais raças. Finalizando, $1,4 \%$ dos RN tiveram alguma malformação congênita.

Tabela 3 - Perfil dos recém-nascidos atendidos no Hospital Universitário de Maringá - Maringá, PR - 2006

\begin{tabular}{|c|c|c|}
\hline Variável & $\mathbf{N}$ & $\%$ \\
\hline \multicolumn{3}{|l|}{ Sexo* } \\
\hline Masculino & 391 & 51,5 \\
\hline Feminino & 368 & 48,5 \\
\hline \multicolumn{3}{|c|}{ Apgar no $1^{\circ} \min * *$} \\
\hline$<7$ & 106 & 14,0 \\
\hline$\geq 7$ & 652 & 86,0 \\
\hline \multicolumn{3}{|c|}{ Apgar no $5^{\circ} \mathrm{min}$} \\
\hline$<7$ & 29 & 3,9 \\
\hline$\geq 7$ & 731 & 96,1 \\
\hline \multicolumn{3}{|l|}{ Raça/cor } \\
\hline Branca & 583 & 76,7 \\
\hline Preta & 7 & 0,9 \\
\hline Parda & 167 & 22,0 \\
\hline Outras & 3 & 0,4 \\
\hline \multicolumn{3}{|l|}{ Peso } \\
\hline$<2.500 \mathrm{~g}$ & 179 & 23,6 \\
\hline$\geq 2.500 \mathrm{~g}$ & 581 & 76,4 \\
\hline \multicolumn{3}{|c|}{ Malformação congênita } \\
\hline Sim & 11 & 1,4 \\
\hline Não & 749 & 98,6 \\
\hline
\end{tabular}

A Figura 1 mostra que o perfil das parturientes e seus RN com município de residência da mãe fora de Maringá está associada ao número de consultas de pré-natal inadequadas, ao baixo peso do RN e ao parto cesárea. O perfil com município de residência da mãe em Maringá está associado ao número de consultas de pré-natal adequadas, ao peso normal do RN e ao parto normal.

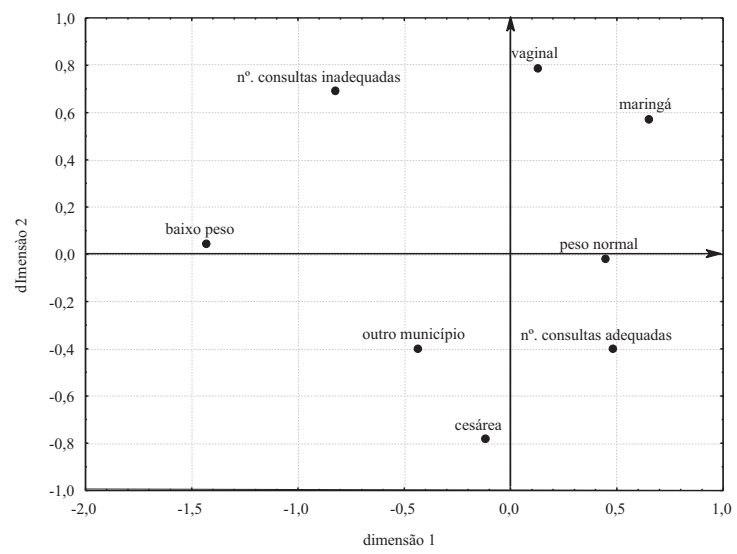

Figura 1 - Mapa de correspondência entre as variáveis município de residência da mãe, tipo de parto, peso ao nascer e número de consultas pré-natal - Maringá, PR - 2006 


\section{DISCUSSÃO}

O número de nascidos vivos no HUM, em 2006, manteve-se próximo da média anual dos nascidos vivos dos últimos seis anos, que foi de 727 nascidos vivos/ano. Este resultado é sugerido pelo número de leitos obstétricos (três leitos de pré-parto e nove leitos de puerpério) e pelo hospital servir de referência para gestação de risco, e o número de nascimentos tornando-se mais restrito. $O$ estudo realizado, em Belo Horizonte, não verificou diferença significante no perfil dos nascidos vivos entre os anos 1992/1994 e a outros municípios do mesmo porte ${ }^{(8)}$, ou seja, o constante perfil dos nascimentos no estudo referenciado, pode indicar nenhuma diferença no perfil dos nascidos vivos nos últimos anos do HUM.

Após 14 anos de implantação do SINASC, no município de Maringá, é observada excelente qualidade no preenchimento da DNV no Hospital Universitário de Maringá, considerando-se fonte de informações adequada para o estudo. As variáveis sem informação para todo o município de Maringá no ano de $1994^{(2)}$ variavam de $0,4 \%$ para tipo de parto, 3,2\% para escolaridade da mãe, 0,1\% para sexo do RN e a variável ocupação da mãe não foi apresentada no trabalho referenciado, pois ainda não fazia parte da DNV. O melhor preenchimento da DNV pode ser pelo seu tempo de implantação, sendo esclarecidas as dúvidas em relação ao preenchimento, como também a conscientização por parte dos serviços de saúde em preencher este documento.

Os percentuais apresentados, na faixa etária das parturientes, foram próximos ao estudo realizado em Belo Horizonte $^{(8)}$. Embora a maioria das parturientes estivesse na faixa etária adequada para a gestação, o alto percentual de mães adolescentes $(27,1 \%)$ indica a necessidade de maiores estratégias de planejamento familiar para esta faixa etária. O resultado de gestação, na adolescência, foi próximo ao encontrado no estudo em São Luís, Estado do Maranhão (1997-1998) com 29,4\%(9), porém mais alto que o encontrado para o Estado do Paraná, em 2005, que foi de $20,0 \%{ }^{(1)}$.

A condição da maioria das parturientes não terem companheiro também foi encontrada em Campinas-2001, e este estudo complementava que as adolescentes sem companheiro foram as que mais apresentaram risco para gravidez ${ }^{(10)}$. Há de se concordar com o estudo divulgado, que a atual configuração da DNV limita a real situação conjugal das parturientes, pois esta variável está categorizada em (solteira, casada, viúva e separada judicialmente) o que impõe a classificação de parturientes com companheiro em união consensual na condição de solteira e não como casadas que seria a classificação mais adequada. A importância da adequada classificação desta variável pode ser pautada no possível apoio econômico de uma situação estável como também pelo apoio psicossocial de se ter um companheiro para dividir este momento de vida.
A escolaridade da parturiente pode ser vista como indicador de condição social, e o maior grau de instrução facilita acesso a emprego e melhoria da posição socioeconômica da família. O percentual de apenas $9,4 \%$ com igual ou maior a 12 anos de estudo está abaixo do encontrado para o Estado do Paraná, em $2005^{(1)}$, que foi de $16,9 \%$. 0 percentual próximo ao analfabetismo, menor ou igual a três anos de estudo (27,0\%) esteve mais elevado que o encontrado para o Estado do Paraná e para o município de Maringá(1) $^{(1)}$ que foi respectivamente de 9,5\% e 1,9\%. A concentração da pouca escolaridade das parturientes apresenta a população esperada do SUS, portanto sem opções para busca de atendimento e convivendo com a realidade das filas de espera.

A ocupação remunerada de apenas 33,5\% complementa os dados anteriores, ou seja, pouca escolaridade, consequentemente, pouca opção socioeconômica. Chama atenção dentre as parturientes que não tinham ocupação remunerada, pois apenas 3,3\% estudavam, ou seja, quantidade expressiva de mulheres jovens. Esta foi a variável com menor preenchimento, a ausência de registro pode ser explicada pela indisponibilidade da Classificação Brasileira de Ocupação - CBO nos serviços, pois em alguns casos esta variável até foi preenchida na DNV, porém fora das opções do CBO, ficando registrada no SINASC como em branco. O não-preenchimento da variável ocupação pode interferir nos estudos socioeconômicos, que para este estudo é necessária a entrega de um CBO em cada local referenciado para o preenchimento da DNV, para evitar o mínimo de casos mal preenchidos. Em outro estudo realizado no Hospital Universitário de Gaffrée e Guinle (UNI-RIO), no município do Rio de Janeiro com gestantes atendidas no Ambulatório de Obstetrícia ${ }^{(5)}$, foram encontrados resultados diferentes em relação à ocupação, $62,7 \%$ tinham alguma ocupação remunerada e apenas $37,2 \%$ não tinham nenhuma ocupação remunerada, porém conforme o resultado do presente estudo, as mães sem ocupação remunerada também eram as donas de casa e estudantes. Com a introdução da opção união consentida na variável estado civil, poderá, em estudos futuros com a variável ocupação, fazer uma estimativa da renda familiar das mães casadas sem ocupação remunerada.

O fato de a maioria da população estudada residir fora de Maringá reforça a característica da clientela que busca o hospital por servir de referência para vários outros municípios pelo seu atendimento às gestações e partos de alto risco, o que explica de modo geral a grande diferença das proporções de indicadores do hospital em relação ao município. Embora a população atendida no HUM resida em municípios diferentes, foi expressiva a utilização do HUM pelos municípios vizinhos, em especial Sarandi e Paiçandu.

Quanto à variável paridade, no estudo realizado em Caxias do Sul, Estado do Rio Grande do Sul, foi demonstrada associação significativa entre esta variável com a qualidade da atenção pré-natal, indicando que quanto maior o número de filhos, mais tardiamente a gestante iniciou o 
acompanhamento do pré-natal e foi observado o número de consultas menor ${ }^{(11)}$. Com uma análise estatística mais aprofundada poder-se-ia aplicar este resultado ao presente estudo, que apresentou na descrição dos dados pouca diferença entre as primíparas $(50,4 \%)$ e as multíparas $(49,6 \%)$, e no número de consultas de pré-natal a maioria $(63,6 \%)$ realizaram sete ou mais consultas de pré-natal.

Referente ao não ter filhos nascidos mortos, foi verificado no estudo realizado no Rio de Janeiro, entre idades mais jovens a não-ocorrência prévia de natimortos. Ou seja, as adolescentes apresentaram uma razão de chance 2,6\% maior de não ter tido filhos mortos quando comparadas às demais mulheres ${ }^{(12)}$. Deste modo, a presença de $27,1 \%$ de parturientes adolescentes, neste estudo, pode indicar o pequeno resultado de não ter filhos nascidos mortos. Considerando que $50,4 \%$ das parturientes eram primíparas e $27,1 \%$ eram adolescentes, indica-se a necessidade de um trabalho efetivo da equipe de saúde no período do parto para propiciar redução na morbimortalidade materna, fetal e neonatal.

A duração da gestação no município de Maringá, em 2004, era de 93,3\% a termo, e somente 4,4\% pré-termos ${ }^{(2)}$. Para o ano de 2006, foi encontrado $73,8 \%$ a termo e $25,5 \%$ pré-termo. A mudança nessas proporções pode esclarecer o aumento para 23,6\% dos RN baixo peso para o ano de 2006, pois no estudo citado, os RN pré-termos foram os que com maior frequência (64,8\%) apresentaram baixo peso ao nascer. De acordo com o Ministério da Saúde, a ocorrência de baixo peso ao nascer expressa restrição do crescimento intra-uterino ou prematuridade ${ }^{(13)}$.

O número de consultas pré-natal pode subsidiar a qualidade da assistência pré-natal, visto que, dentre os vários fatores que levam à prematuridade, o número de nascidos vivos com baixo peso e óbitos, por afecções do período perinatal, poderia ser evitado com acompanhamento pré-natal satisfatório(8,14). Embora neste estudo tenha sido observado $36,2 \%$ de mulheres com menos de sete consultas, em outro estudo(15), 41,2\% não tiveram nem as seis consultas pré-natal, conforme preconizado pelo Ministério da Saúde ${ }^{(13)}$. Embora o número de consultas de pré-natal possa ser utilizado para analisar a cobertura dos serviços de pré-natal, subsidiando o planejamento e avaliação do atendimento pré-natal tem como limitações não abranger as gestantes que tiveram aborto ou um filho nascido morto e não reflete na qualidade do atendimento prestado nas consultas de pré-natal.

O índice 63,2\%, referente ao adequado número de consultas pré-natal reforçado pela maioria das parturientes $(59,3 \%)$ ser de outros municípios, parece demonstrar que nos municípios vizinhos a Maringá há dificuldade em prestar atendimento às gestantes em geral, especialmente às consideradas de risco. Desse modo, são encaminhadas para o HUM, que está referenciado para gestação e parto de alto risco e possui unidade de terapia intensiva adulta e neonatal. Um pré-natal adequado constitui forma de medicina preventiva, pois disponibiliza monitoramento adequado, solicitação de exames, vacinas, e orientações específicas a fim de diminuir a ansiedade da gestante e, possibilita detectar o diagnóstico precoce de patologias que podem ser tratadas em tempo hábil, contribuindo na queda da mortalidade infantil e materna.

Como descrito na literatura, o Brasil de modo geral tem taxas de cesáreas superiores ao preconizado pelo Ministério da Saúde ${ }^{(13)}$, que seriam de $40,0 \%$ para alto risco e de $25,0 \%$ para risco habitual. O resultado deste estudo não foi diferente apresentando $50,4 \%$ de partos cesárea, que no entender do HUM, este percentual é referência regional ao que se refere à gravidez de alto risco. Porém, os valores são maiores aos encontrados no Instituto Materno Infantil no Estado de Pernambuco (35\%) ${ }^{(16)}$, hospital de referência para gestação de risco às usuárias do SUS. A tendência nos índices de cesáreas reafirma o uso abusivo da tecnologia associado à realização de procedimentos desnecessários, a precariedade na qualidade da assistência prénatal e parto e a deficiência da formação e qualificação dos profissionais.

No HUM, em $1994^{(2)}$, a maioria dos RN $(54,4 \%)$ havia nascido com peso igual ou maior a $3.000 \mathrm{~kg}$ e a proporção de baixo peso era de $14,8 \%$. Os resultados apontavam para o fato de o HUM ser o único hospital público do município, servir de referência para a região, e concentrava gestantes de alto risco. Alguns estudos ${ }^{(8,12-13,15)}$ que investigaram a prevalência de baixo peso variaram suas proporções de 9,8\% até 18,7\%. Estes dados demonstram que o HUM apresenta incidências elevadas de baixo peso entre os recém-nascidos, quando comparadas com outras cidades do Brasil. A comparação dos resultados de recém-nascidos de baixo peso com a de outros estudos, faz-nos refletir sobre os fatores condicionantes para ter filhos com peso inadequado. É possível que exista deficiência no controle pré-natal, seja na cobertura ou na qualidade do atendimento, ou relação com outros fatores de risco como a prematuridade e a idade das mães nos grupos extremos de vida reprodutiva ${ }^{(12,14)}$.

Os resultados para as variáveis tipo de gravidez, sexo, raça/cor e índice de Apgar do RN neste estudo foram compatíveis segundo a outros estudos e a realidade local do município descrito a seguir. Em relação ao tipo de gravidez, alguns estudos ${ }^{(8,12)}$ encontraram a maioria dos casos $(98,2 \%)$ com gestação única. A prevalência para nascidos do sexo masculino, também foi encontrada para o município de Maringá em 1994, para o Estado do Paraná e Brasil(1-2). O Paraná é um Estado com prevalência de população bran$\mathrm{ca}^{(1)}$. No ano de 2005 , a maioria dos nascimentos $(93,2 \%)$ referiu como de raça/cor branca, condizendo com a maioria dos nascidos no HUM. Os resultados do índice de Apgar, neste estudo, foram próximos a outros estudos ${ }^{(12,16-17)}$. 
A proporção de malformações congênitas foi maior ao proporcional 0,6\% de ocorrência em Maringá e no Estado do Paraná, em 2005, fato já esperado pela referência de gestação de risco. Tendo presente a elevada prevalência das malformações congênitas algum grau de rastreamento há de ser estendido a toda população de grávidas ${ }^{(7)}$. 0 impacto dos defeitos congênitos no Brasil vem aumentando progressivamente, tendo passado da quinta para a segunda causa dos óbitos em menores de um ano entre 1980 e 2000, apontando para a necessidade de estratégias específicas na política de saúde ${ }^{(18)}$.

A associação entre os nascimentos de mães residentes em Maringá, número de consultas de pré-natal adequadas, parto vaginal e peso normal, demonstrou a procura ao HUM pelas mães de baixo risco conforme é referência para o município. Já os nascimentos de mães residentes fora de Maringá associou-se a número de consultas de pré-natal inadequadas, parto cesárea e baixo peso, apresentando a procura pelas mães de alto risco conforme o HUM é referência para os outros municípios. A associação entre as variáveis indicou que o HUM está sendo referência conforme estabelecido entre os municípios e, também pode sugerir a necessidade de melhoria nas pactuações dos indicadores nos municípios fora de Maringá, pois foram os que resultaram nos piores indicadores para parto cesárea e baixo peso. Para número de consultas de pré-natal provavelmente os municípios fora de Maringá não apresentavam número de consultas de pré-natal $\geq 7$, que de acordo com as instruções dos indicadores para a pactuação é específica para os municípios em que a proporção de nascidos vivos de mães de quatro ou mais consultas de pré-natal superou $90 \%$ do ano anterior, situação foi encontrada no HUM $(91,1 \%)$ provavelmente advinda das mães residentes em Maringá.

\section{CONCLUSÕES}

Com os resultados deste estudo, foi possível comparar a realidade do perfil das parturientes e dos seus RN atendidos no HUM com outras realidades do Brasil. O resultado dos indicadores referenciados apresentou situações de perigo na sobrevivência do grupo populacional estudado, quando comparado com o município de Maringá e o Estado do Paraná, principalmente pelo elevado número de parturientes adolescentes, com baixa escolaridade, residência fora do município de atendimento ao parto, prematuridade e baixo peso reforçando a referência para o atendimento de parto de risco neste hospital e o perfil do binômio mãefilho, usuários esperados nos serviços do SUS.

A identificação de situações de perigo, na sobrevivência dos recém-nascidos como resultado do sistema de saú- de e diferenças no acesso, e a qualidade da assistência estão intimamente relacionadas com o desempenho dos serviços de saúde, o que chama à responsabilidade dos gestores no sentido de proverem acesso oportuno a serviços de qualidade para a população. Portanto, a regionalização e qualificação da assistência e a universalização do acesso mostram-se como ações prioritárias e intervenções efetivas para a diminuição das situações de perigo na sobrevivência dos recém-nascidos na 15르.

Foi comprovada a possibilidade de utilização do SINASC como ótimo instrumento para avaliação e planejamento da saúde materno-infantil e, portanto, a viabilização de estudos oportunos para a melhoria das condições de saúde materno-infantil. Entretanto, ainda é ressaltado que para haver vigilância e controle dos indicadores de saúde é necessário investimento na melhoria da qualidade da informação, tanto no preenchimento das DNV, quanto na fidedignidade e confiabilidade dos dados. Revisões periódicas com os responsáveis pelo preenchimento da DNV e do SINASC são imprescindíveis para verificar as dificuldades no preenchimento e assim permitir possibilidades de melhoria na fidedignidade das informações de acordo com a realidade.

As condições de nascimento do RN podem estar relacionadas à qualidade do atendimento à mulher durante a gravidez, trabalho de parto e parto. As informações obtidas, neste estudo, poderão assegurar assistência ao parto e nascimento em condições seguras de infra-estrutura material e recursos humanos, com trabalho em equipe interdisciplinar com profissionais treinados e integrados para atender a situações rotineiras e emergenciais da instituição de ensino como a realidade apresentada.

A maioria das parturientes ter referido a realização de mais de sete consultas de pré-natal leva à reflexão para um estudo complementar, pelo seguinte questionamento: realizaram as consultas de pré-natal por busca ativa dos serviços de atendimento pré-natal ou foi pela gestação de alto risco que necessitou de mais consultas?

Finalizando, acredita-se que para melhor atenção da saúde materno-infantil é necessário envolvimento dos profissionais da saúde na descentralização dos serviços de saúde. Com a regionalização dos serviços de saúde, é possível articular e/ou mobilizar ações de melhoria na saúde da população de acordo com a especificidade de cada região com consequente promoção de intervenções efetivas nos diversos níveis de atuação. E a melhoria na pactuação dos indicadores entre os municípios poderá refletir nas necessidades de saúde de cada população na busca da equidade social.

\section{REFERÊNCIAS}

1. Brasil. Ministério da Saúde. DATASUS: informações de saúde [texto na Internet]. Brasília; 2002. [citado 2007 jul. 15]. Disponível em: http://www.saude.datasus.gov.br
2. Carvalho WO. Nascidos vivos e óbitos perinatais dos municípios de Maringá, Paiçandu e Sarandi, PR-1994 [dissertação]. Londrina: Universidade Estadual de Londrina; 1996. 
3. Brasil. Ministério da Saúde. Fundação Nacional de Saúde. Manual de procedimentos do sistema de informações sobre nascidos vivos. Brasília; 2001.

4. Okazaki, ELFJ. Perfil biológico e social das parturientes do Hospital Geral do Grajaú [dissertação]. Santo Paulo: Faculdade de Medicina, Universidade de Santo Amaro; 2002.

5. Spindola T, Penna LHG, Progianti JM. Perfil epidemiológico de mulheres atendidas na consulta pré-natal de um hospital universitário. Rev Esc Enferm USP. 2006;40(3):381-8.

6. Machado SP, Kuchenbeck R. Desafios e perspectivas futuras dos hospitais universitários no Brasil. Ciênc Saúde Coletiva. 2007;12(4):871-7.

7. Rezende J, Montenegro CAB. Obstetrícia fundamental. Rio de Janeiro: Guanabara Koogan; 2006.

8. Rodrigues CS, Magalhães Júnior HM, Evangelista PA, Ladeira RM, Laudares S. Perfil dos nascidos vivos no município de Belo Horizonte, 1992-1994. Cad Saúde Pública. 1997;13(1):53-7.

9. Simões VMF, Silva AAM, Bettiol H, Lamy Filho F, Tonial SR, Mochel EG. Características da gravidez na adolescência em São Luís, Maranhão. Rev Saúde Pública. 2003;37(5):559-65.

10. Carniel EF, Zanolli ML, Almeida CAA, Morcillo AM. Características das mães adolescentes e de seus recém-nascidos e fatores de risco para gravidez na adolescência em Campinas, SP, Brasil Rev Bras Saúde Matern Infatil. 2006;6(4):419-26.
11. Trevisan MR, Lorenzi DRS, Araújo NM, Ésber K. Perfil da assistência pré-natal entre usuárias do Sistema Único de Saúde em Caxias do Sul. Rev Bras Ginecol Obstet. 2002;24(5):293-9.

12. Pastrana VV. Estudo epidemiológico das gestantes adolescentes de Niterói [dissertação]. Rio de Janeiro: Escola Nacional de Saúde Pública, Fundação Oswaldo Cruz;1998.

13. Brasil. Ministério da Saúde. Portaria n 493, de 13 de março de 2006. Pacto de Indicadores da Atenção Básica, 2006. Brasília; 2006.

14. Kilsztajn S, Rossbach A, Carmo MSN, Sugahara GTL. Assistência pré-natal, baixo peso e prematuridade no Estado de São Paulo, 2000. Rev Saúde Pública. 2003;37(3):303-10.

15. Domingues RMSM, Santos EM, Leal MC. Aspectos da satisfação das mulheres com a assistência ao parto: contribuição para o debate. Cad Saúde Pública. 2004;20 Sup 1:S52-S62.

16.Vidal SA, Arruda BKG, Vanderlei LC, Frias PG. Avaliação da série histórica dos nascidos vivos em unidade terciária de Pernambuco-1991 a 2000. Rev Assoc Med Bras.2005;51(1):17-22.

17. Horovitz DDG, Llerena Junior JC, Mattos RA. Atenção aos defeitos congênitos no Brasil: panorama atual. Cad Saúde Pública. 2005;21(4):1055-64.

18. Fernandes K, Kimura AF. Práticas assistenciais em reanimação do recém-nascido no contexto de um centro de parto normal. Rev Esc Enferm USP. 2005;39(4):383-90. 